\title{
Relationship between body-roundness index and metabolic syndrome in type 2 diabetes
}

This article was published in the following Dove Press journal:

Diabetes, Metabolic Syndrome and Obesity: Targets and Therapy

\section{Bowei Liu \\ Bo Liu \\ Guangfei Wu \\ Fuzai Yin}

Department of Endocrinology, First Hospital of Qinhuangdao, Qinhuangdao 066000, Hebei Province, China
Correspondence: Fuzai Yin Department of Endocrinology, First Hospital of Qinhuangdao, 258 Wenhua Road, Qinhuangdao, Hebei 066000, China Tel +863355908603

Fax +863353032042

Email yinfu_zai@I63.com
Background: The present study evaluated the relationship between body-roundness index (BRI) and metabolic syndrome (MetS) in type 2 diabetes.

Methods: A cross-sectional study was conducted on 585 type 2 diabetes in Qinhuangdao. MetS was defined as per the Chinese Diabetes Society.

Results: The BRI was strongly associated with the odds of having MetS in both males and females after adjustment for age, history of diabetes, and body-mass index $(P<0.05)$. AUC of the BRI were 0.824 for males and 0.775 for females $(P<0.001)$. The optimal cutoff points for the BRI were 3.85 in males (sensitivity $76.5 \%$, specificity $82.1 \%$ ) and 4.05 in females (sensitivity $76.4 \%$, specificity $70.3 \%$ ).

Conclusion: The BRI was correlated with MetS and was an effective indicator for the screening of MetS in type 2 diabetes.

Keywords: body-roundness index, metabolic syndrome, type 2 diabetes

\section{Introduction}

Diabetes is a growing global problem. According to the International Diabetes Federation, $8.8 \%$ of adults aged $20-79$ years were estimated to have diabetes in $2017 .{ }^{1}$ Diabetes leads to increased risk of cardiovascular complications. In turn, cardiovascular diseases is a major cause of death in patients with diabetes. ${ }^{2}$ Metabolic syndrome (MetS) is a cluster of cardiometabolic risk factors. The prevalence of MetS is high ${ }^{3}$ and further increases the risk of cardiovascular diseases in type 2 diabetes. ${ }^{4-6}$ Early identification and appropriate treatment canreduce the excess risk of MetS.

Central obesity is at the core of MetS. Therefore, simple obesity indices are used for screening MetS. Waistcircumference (WC) and waist:height ratio (WHtR) are the most widely used indices. In recent years, some new indices based on WC have been proposed. Visceral adiposity index, lipid-accumulation product, and $\mathrm{WC}$-triglyceride (TG) index were calculated with WC, TG, and high-density-lipoprotein cholesterol (HDL-C). ${ }^{7}$ Body-shape index, conicity index, and abdominal volume index were calculated with WC, height, weight, and hip circumference. ${ }^{8-10}$

The body-roundness index (BRI) was proposed by Thomas et al in $2013 .{ }^{11}$ It is an index estimated with the use of height, weight, WC, and hip circumference (optional). The web-based calculator gives a result for BRI and streamlines the calculation. This method is derived from geometry and provides body-type location relative to a healthy body. BRI shows a superior predictive power for MetS in the general population, obese and overweight population, and postmenopausal women. ${ }^{12-14}$ 
Cutoff points for the BRI are not unified across different populations. ${ }^{12,15,16}$ The BRI is closely associated with diabetes, and has been used to identify diabetes in a crosssectional study. ${ }^{17,18}$ It can also predict diabetes. ${ }^{19}$ To our knowledge, no investigators have attempted to use the BRI for screening MetS in type 2 diabetes. Therefore, it was necessary to explore cutoff points for the BRI in type 2 diabetes. The aim of our study was to evaluate the relationship between the BRI and MetS in type 2 diabetes.

\section{Methods}

\section{Subjects}

After informed consent had been obtained, a crosssectional study was conducted. All subjects were patients with type 2 diabetes $>18$ years old. ${ }^{20}$ Exclusion criteria were type 1 diabetes, clinical evidence of other endocrinopathy, pregnacy, and acute or chronic inflammation. This study was approved by the ethics committee of the First Hospital of Qinhuangdao. All subjects provided written informed consent before study initiation. This study was conducted in accordance with the Declaration of Helsinki.

\section{Measurements}

Anthropometric measurements, including height, weight, $\mathrm{WC}$, and blood pressure, were obtained. WC was measured midway between the lowest rib and the top of the iliac crest. BRI was calculated with an online calculator (http://www.pbrc.edu/bodyroundness). ${ }^{11}$ The formula was $\mathrm{BRI}=364.2-365.5 \times\left(1-[\mathrm{WC} / 2 \pi]^{2} /\left[0.5 \times \text { height }^{2}\right)^{1 / 2}\right.$.

After a 10-hour overnight fast, blood samples were collected from an antecubital vein into heparinized tubes. Fasting plasma glucose (FPG) concentrations and serumlipid levels were measured using enzymatic assays with an autoanalyzer (Hitachi, Tokyo, Japan).

\section{Definition of metabolic syndrome}

MetS was defined as per the Chinese Diabetes Society. Participants had to meet any three or more of fivefactors: 1) $\mathrm{WC} \geq 90 \mathrm{~cm}$ (male) and $85 \mathrm{~cm}$ (female), 2) $\mathrm{FPG} \geq 6.1 \mathrm{mmol} / \mathrm{L}$ or 2-hour PG levels $\geq 7.8 \mathrm{mmol} / \mathrm{L}$ after a $75 \mathrm{~g}$ oral glucosetolerance test or have been diagnosed with diabetes, 3) blood pressure $\geq 130 / 85 \mathrm{mmHg}$ or had been diagnosed with hypertension, 4) $\mathrm{TG} \geq 1.7 \mathrm{mmol} / \mathrm{L}$, and 5) HDL-C $<1.04 \mathrm{mmol} / \mathrm{L}^{21}$

\section{Statistical analyses}

All analyses were performed using SPSS 11.5 (SPSS, Chicago, IL, USA). Comparisons were conducted between groups using independent samples $t$-test and $\chi^{2}$ analysis. For BRI, we divided them into increasing sex-specific tertiles and used multiple logistic regression analysis to calculate ORs for MetS across tertiles, with tertile 1 as the reference group, adjusting for age, history of diabetes, and body-mass index (BMI). ROC curves for BRI were drawn to show how well they were able to separate subjects into groups with or without MetS. Comparisons of AUCs were performed with MedCalc 11.4.2.0 (Ostend, Belgium). $P<0.05$ was considered statistically significant.

\section{Results}

This study enrolled 585 type 2 diabetes patients (339 males and 246 females, age $54.5 \pm 12.5$ years). Average history of diabetes was $6.4 \pm 6.7$ years. Prevalence of MetS was similar between males and females (males $75.2 \%$ vs females $74.0 \%, \chi^{2}=0.116$; $P=0.734)$. Characteristics of the study population are presented in Table 1. Age, history of diabetes, and FPG between the two groups were similar $(P>0.05)$. BMI, WC, WHtR, BRI, systolic blood pressure, diastolic blood pressure, and TG were all significantly higher in the MetS group than the non-MetS group $(P<0.001)$. HDL-C was significantly lower in the MetS group than the non-MetS group $(P<0.001)$.

BRI was strongly associated with the odds of having MetS in both males and females, after adjustment for age, history of diabetes, and BMI $(P<0.001)$. In males, patients in BRI tertile 2 (OR 5.035, $P<0.001$ ) and tertile 3 (OR 7.195, $P<0.001)$ were more likely to have MetS than those in tertile 1. In females, patients in BRI tertile 2 (OR 4.616, $P<0.001$ ) and tertile 3 (OR 3.772, $P=0.012$ ) were more likely to have MetS than those in tertile 1 (Table 2)

AUCs for WC were 0.842 for males and 0.798 for females $(P<0.001)$ for WHtR 0.825 for males and 0.775 for females $(P<0.001)$, and for BRI 0.824 for males and 0.775 for females $(P<0.001$; Figure 1$)$, while they were similar among BRI, WC and WHtR $(P>0.05$; Table 3$)$. Optimal cutoff points for BRI were 3.85 in males (sensitivity $76.5 \%$, specificity $82.1 \%$ ) and 4.05 in females (sensitivity $76.4 \%$, specificity $70.3 \%$ ).

\section{Discussion}

Our study presents evidence that the BRI may be a useful tool for thescreening of MetS in type 2 diabetes, but not superior to $\mathrm{WC}$ and WHtR. Zhang et al found that BRI showed similar ability to detect subjects with MetS among general population. ${ }^{12}$ Similar results have also been observed in people with intermediate cardiovascular risk, postmenopausal women, and obese and overweight adults. ${ }^{13-15}$ The prevalence 
Table I Basic characteristics of study population

\begin{tabular}{|c|c|c|c|c|}
\hline Variables & Non-MetS group $(n=\mid 48)$ & MetS group $(n=437)$ & $t$ or $\chi^{2}$ & $P$ \\
\hline Males/females & $84 / 64$ & $255 / 182$ & 0.116 & 0.734 \\
\hline Age (years) & $53.7 \pm 12.6$ & $54.8 \pm 12.5$ & 0.876 & 0.381 \\
\hline History of diabetes (years) & $6.3 \pm 6.4$ & $6.4 \pm 6.8$ & 0.066 & 0.948 \\
\hline BMI $\left(\mathrm{kg} / \mathrm{m}^{2}\right)$ & $23.9 \pm 3.5$ & $27.1 \pm 3.3$ & 9.865 & $<0.001$ \\
\hline WC $(\mathrm{cm})$ & $84.2 \pm 8.6$ & $95.1 \pm 10.0$ & 12.685 & $<0.001$ \\
\hline WHtR & $0.50 \pm 0.05$ & $0.57 \pm 0.05$ & 11.128 & $<0.001$ \\
\hline BRI & $3.5 \pm 1.0$ & $4.7 \pm 1.3$ & 11.486 & $<0.001$ \\
\hline $\mathrm{SBP}(\mathrm{mmHg})$ & $123.6 \pm 15.3$ & $|3| .7 \pm 18.8$ & 5.243 & $<0.001$ \\
\hline $\mathrm{DBP}(\mathrm{mmHg})$ & $79.4 \pm 9.1$ & $83.2 \pm 10.0$ & 4.189 & $<0.001$ \\
\hline FPG (mmol/L) & $9.07 \pm 4.01$ & $9.13 \pm 3.44$ & 0.149 & 0.882 \\
\hline TG (mmol/L) & $1.15 \pm 0.54$ & $2.86 \pm 2.60$ & $|2.87|$ & $<0.001$ \\
\hline HDL-C (mmol/L) & $1.31 \pm 0.26$ & $1.05 \pm 0.30$ & 9.257 & $<0.001$ \\
\hline
\end{tabular}

Abbreviations: MetS, metabolic syndrome; BMI, body-mass index; WC, waist circumference; WHtR, waist:height ratio; BRI, body-roundness index; SBP, systolic blood pressure; DBP, diastolic blood pressure; FPG, fasting plasma glucose; TG, triglyceride; HDL-C, high-density-lipoprotein cholesterol.

Table 2 Prevalence of metabolic syndrome according to the level of body-roundness index

\begin{tabular}{|c|c|c|c|c|c|c|c|}
\hline Men $(n=339)$ & n (\%) & OR (95\%Cl) & $P$ & Women $(n=246)$ & n (\%) & OR (95\%CI) & $P$ \\
\hline Tertiles I $(n=|| 3)$ & $52(46.0)$ & I & & Tertiles I $(n=82)$ & $39(47.6)$ & I & \\
\hline Tertiles $2(n=|| 3)$ & $96(85.0)$ & $5.035(2.560-9.904)$ & $<0.001$ & Tertiles $2(n=82)$ & $70(85.4)$ & $4.616(2.056-10.363)$ & $<0.001$ \\
\hline Tertiles $3(n=\mid 13)$ & $107(94.7)$ & $7.195(2.576-20.099)$ & $<0.001$ & Tertiles $3(n=82)$ & $73(89.0)$ & $3.772(1.345-10.577)$ & 0.012 \\
\hline
\end{tabular}

Note: Multiple logistic regression analysis, adjustment for age, history of diabetes, and body-mass index.
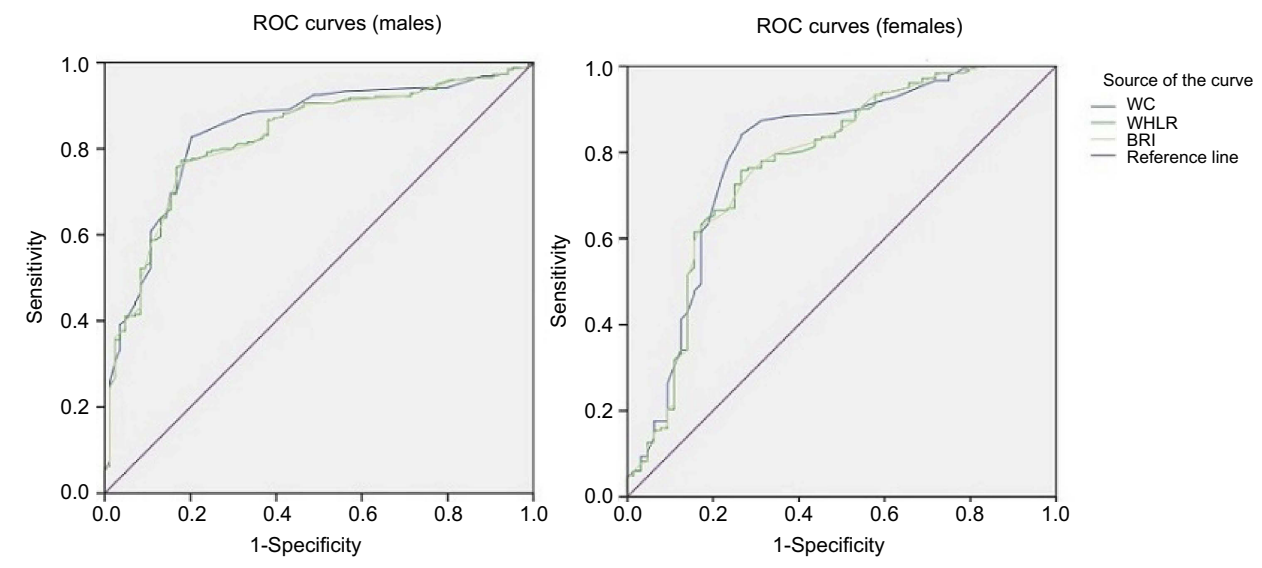

Figure I ROC curves of obesity indices used to identify the presence of metabolic syndrome in type 2 diabetes.

Abbreviations: WC, waist circumference; WHtR, waist:height ratio.

of MetS was $28.3 \%-55.7 \%$ in these studies. Type 2 diabetes is often accompanied by MetS. ${ }^{22}$ Prevalence was $>70 \%$ in our study, higher than previous research, but the power of BRI was similar to previous research.

Cutoff points for the BRI have not been consistent across studies: $3.47-5.69$ for males and 3.18-5.77 for females. ${ }^{12,15,16}$ This difference could be because of study populations, race, and diagnostic criteria for MetS. In our study, cutoff points for BRI were 3.85 for males and 4.05 for females in type 2 diabetes, similar to Chinese studies and lower than a Spanish study.

The capacity of the BRI to predict each component of MetS, such as dyslipidemia, hypertension, and diabetes, were comparable but not superior to the classical indicators of obesity. ${ }^{18,23-25}$ Insulin resistance and chronic inflammation are the main mechanisms of MetS. BRI showed significant correlation with insulin resistance and inflammatory factors and equivalence with traditional obesity indices. ${ }^{13,26,27}$ 
Table 3 AUC and SE of each variable for screening of metabolic syndrome in type 2 diabetes

\begin{tabular}{|l|l|l|l|l|l|l|}
\hline Variables & AUC $(\mathbf{9 5} \% \mathbf{C I})$ & SE & $\boldsymbol{P}$ & $Z^{*}$ & $P^{*}$ & $Z^{\#}$ \\
\hline Men & & & & & & \\
WC & $0.842(0.794-0.89 I)$ & 0.025 & $<0.001$ & & & \\
WHtR & $0.825(0.776-0.875)$ & 0.025 & $<0.001$ & 0.481 & 0.630 & \\
BRI & $0.824(0.775-0.874)$ & 0.025 & $<0.001$ & 0.509 & 0.610 & 0.028 \\
\hline Women & & & & & & \\
WC & $0.798(0.726-0.870)$ & 0.037 & $<0.001$ & & & \\
WHtR & $0.775(0.702-0.848)$ & 0.037 & $<0.001$ & 0.440 & 0.660 & \\
BRI & $0.775(0.702-0.848)$ & 0.037 & $<0.001$ & 0.440 & 0.660 & 0 \\
\hline
\end{tabular}

Notes: *Compared with WC; ${ }^{\#}$ compared with WHtR. AUCs were similar among WC, WHtR, and BRI $(P>0.05)$.

Abbreviations: WC, waist circumference; $\mathrm{WHtR}$, waist:height ratio; $\mathrm{BRI}$, body-roundness index.

There are limitations to our study. First, although hip circumference was not a mandatory variable for BRI, combined hip circumference and WC can enhanced the performance of the BRI. ${ }^{11}$ There were not hip-circumference data in our database. Second, patients were recruited from a single hospital, and replicability of these results should be tested with a multicenter study. Third, other confounding factors, such as physical activity, diet, and other lifestyle factors, were not analyzed in our study. Fourth, the sample was relatively small. Studies with larger samples are necessary in future.

In summary, BRI was correlated with MetS and an effective indicator for the screening of MetS in type 2 dabetes; however, it showed no advantage over traditional obesiy indices.

\section{Disclosure}

The authors report no conflicts of interest in this work.

\section{References}

1. Federation. ID. Idf Diabetes Atlas. 8th ed. Brussels, Belgium: International Diabetes Federation; 2017. Available from: www.diabetesatlas.org.

2. Federation. ID. Diabetes and Cardiovascular Disease. Brussels, Belgium: International Diabetes Federation; 2016. Available from: www.idf.org/cvd.

3. Udenze IC, Azinge EC, Arikawe AP, et al. The prevalence of metabolic syndrome in persons with type 2 diabetes at the Lagos university tteaching hospital, Lagos, Nigeria. West Afr J Med. 2013;32: 126-132.

4. Guzder RN, Gatling W, Mullee MA, Byrne CD. Impact of metabolic syndrome criteria on cardiovascular disease risk in people with newly diagnosed type 2 diabetes. Diabetologia. 2006;49:49-55. doi:10.1007/ s00125-005-0063-9

5. Bonora E, Targher G, Formentini G, et al. The metabolic syndrome is an independent predictor of cardiovascular disease in type 2 diabetic subjects. prospective data from the verona diabetes complications study. Diabet Med. 2004;21:52-58.

6. Yao MF, He J, Sun X, et al. Gender differences in risks of coronary heart disease and stroke in patients with type 2 diabetes mellitus and their association with metabolic syndrome in China. Int $J$ Endocrinol. 2016;2016:8483405. doi:10.1155/2016/8483405
7. Ma CM, Lu N, Wang R, Liu XL, Lu Q, Yin FZ. Three novel obese indicators perform better in monitoring management of metabolic syndrome in type 2 diabetes. Sci Rep. 2017;7:9843. doi:10.1038/s41598-01710446-3

8. Haghighatdoost F, Sarrafzadegan N, Mohammadifard N, Asgary S, Boshtam M, Azadbakht L. Assessing body shape index as a risk predictor for cardiovascular diseases and metabolic syndrome among Iranian adults. Nutrition. 2014;30:636-644. doi:10.1016/j.nut.2013.10.021

9. Costa EC, Sa JC, Soares EM, Lemos TM, Maranhao TM, Azevedo GD. Anthropometric indices of central obesity how discriminators of metabolic syndrome in Brazilian women with polycystic ovary syndrome. Gynecol Endocrinol. 2012;28:12-15. doi:10.3109/09513590.2011.583956

10. Motamed N, Sohrabi M, Poustchi H, et al. The six obesity indices, which one is more compatible with metabolic syndrome? A population based study. Diabetes Metab Syndr. 2017;11:173-177. doi:10.1016/j.dsx.2016.08.024

11. Thomas DM, Bredlau C, Bosy-Westphal A, et al. Relationships between body roundness with body fat and visceral adipose tissue emerging from a new geometrical model. Obesity (Silver Spring). 2013;21:2264-2271. doi:10.1002/oby.20408

12. Zhang J, Zhu W, Qiu L, Huang L, Fang L. Sex- and age-specific optimal anthropometric indices as screening tools for metabolic syndrome in Chinese adults. Int $J$ Endocrinol. 2018;2018:1067603. doi: $10.1155 / 2018 / 1528437$

13. Li G, Wu HK, Wu XW, et al. The feasibility of two anthropometric indices to identify metabolic syndrome, insulin resistance and inflammatory factors in obese and overweight adults. Nutrition. 2019;57:194-201. doi:10.1016/j.nut.2018.05.004

14. Liu PJ, Ma F, Lou HP, Zhu YN. Body roundness index and body adiposity index: two new anthropometric indices to identify metabolic syndrome among Chinese postmenopausal women. Climacteric. 2016;19:433-439. doi:10.1080/13697137.2016.1202229

15. Gomez-Marcos MA, Gomez-Sanchez L, Patino-Alonso MC, et al. Capacity adiposity indices to identify metabolic syndrome in subjects with intermediate cardiovascular risk (MARK study). PLoS One. 2019;14:e0209992. doi:10.1371/journal.pone.0209992

16. Wang H, Liu A, Zhao T, et al. Comparison of anthropometric indices for predicting the risk of metabolic syndrome and its components in Chinese adults: a prospective, longitudinal study. BMJ Open. 2017;7: e016062. doi:10.1136/bmjopen-2017-016062

17. Zhao Q, Zhang K, Li Y, et al. Capacity of a body shape index and body roundness index to identify diabetes mellitus in han Chinese people in Northeast China: a cross-sectional study. Diabet Med. 2018;35:1580-1587. doi:10.1111/dme.13787

18. Chang Y, Guo X, Chen Y, et al. A body shape index and body roundness index: two new body indices to identify diabetes mellitus among rural populations in northeast China. BMC Public Health. 2015;15:794. doi:10.1186/s12889-015-2150-2 
19. Yang J, Wang F, Wang J, et al. Using different anthropometric indices to assess prediction ability of type 2 diabetes in elderly population: a 5 year prospective study. BMC Geriatr. 2018;18:218. doi:10.1186/s12877-0180912-2

20. American Diabetes A. Diagnosis and classification of diabetes mellitus. Diabetes Care. 2014;37(Suppl 1):S81-90. doi:10.2337/dc14-S081

21. Society CD. Chinese guideline for type 2 diabetes. Chin J Endocrinol Metab. 2014;30:893-942.

22. Foroozanfar ZM, Najafipour HP, Khanjani NPM, Bahrampour APM, Ebrahimi HM. The prevalence of metabolic syndrome according to different criteria and its associated factors in type 2 diabetic patients in Kerman, Iran. Iran J Med Sci. 2015;40:522-525.

23. Zaid M, Ameer F, Munir R, et al. Anthropometric and metabolic indices in assessment of type and severity of dyslipidemia. J Physiol Anthropol. 2017;36:19. doi:10.1186/s40101-017-0134-X

24. Chang Y, Guo X, Guo L, Li Z, Li Y, Sun Y. The feasibility of two new anthropometric indices to identify hypertension in rural China: a cross-sectional study. Medicine (Baltimore). 2016;95:e5301. doi:10.1097/MD.0000000000004864
25. Tian S, Zhang X, Xu Y, Dong $\mathrm{H}$. Feasibility of body roundness index for identifying a clustering of cardiometabolic abnormalities compared to BMI, waist circumference and other anthropometric indices: the China health and nutrition survey, 2008 to 2009. Medicine (Baltimore). 2016;95:e4642. doi:10.1097/ MD.0000000000004864

26. Feng J, He S, Chen X. Body adiposity index and body roundness index in identifying insulin resistance among adults without diabetes. Am J Med Sci. 2019;357:116-123. doi:10.1016/j. amjms.2018.11.006

27. Barazzoni R, Gortan Cappellari G, Semolic A, et al. Central adiposity markers, plasma lipid profile and cardiometabolic risk prediction in overweight-obese individuals. Clin Nutr. 2019;38:1171-1179.
Diabetes, Metabolic Syndrome and Obesity: Targets and Therapy is an international, peer-reviewed open-access journal committed to the rapid publication of the latest laboratory and clinical findings in the fields of diabetes, metabolic syndrome and obesity research. Original research, review, case reports, hypothesis formation, expert opinion and commentaries are all considered for publication. The manuscript management system is completely online and includes a very quick and fair peer-review system, which is all easy to use. Visit http://www.dovepress.com/testimonials.php to read real quotes from published authors. 